\title{
Tumores estromales gastrointestinales (GIST) en el Hospital Universitario del Caribe, Cartagena de Indias (Colombia), 2009-2011: serie de casos
}

\author{
- Eusebio Enrique Contreras Borrego', Haroldo Estrada López²
}

${ }^{1}$ Facultad de Medicina, Universidad de Cartagena.

2 Departamento Médico, Sección de Oncología Clínica.

Introducción. Los tumores estromales gastrointestinales (GIST, por su sigla en inglés) son neoplasias malignas poco frecuentes. El objetivo de este estudio es describir las características clínicas, estudios diagnósticos, aspectos quirúrgicos y seguimiento de pacientes con GIST en el Hospital Universitario del Caribe, Cartagena de Indias, entre los años 2009 y 2011.

Materiales y métodos. Serie de casos. A partir de la base de datos del servicio de patología del Hospital Universitario del Caribe, fueron seleccionados todos los casos diagnosticados en espécimen de resección quirúrgica entre los años 2009 y 2011. Se revisó el historial médico en búsqueda de hallazgos clínicos, imaginológicos, endoscópicos, histopatológicos, tratamiento y seguimiento.

Resultados. Se encontraron 6 casos que corresponden a: 1 esofágico, 2 gástricos, 1 duodenal, 1 yeyunal y 1 colónico. Las edades oscilan entre los 38 y 72 años (promedio: 52). Las manifestaciones clínicas más importantes fueron el síndrome anémico y el dolor abdominal. La ultrasonografía se destaca como estudio diagnóstico inicial. Los estudios histopatológicos de los especímenes de resección quirúrgica reportaron márgenes negativos, inmunohistoquímica CD117(+) y displasia de alto grado. En 2 casos, se realizó resección quirúrgica mínimamente invasiva. El tratamiento clínico fue con imatinib y, en algunos casos, se reporta recurrencia tumoral.

Conclusión. Los hallazgos en cada caso son acordes con la literatura. Existen controversias y la experiencia médica es limitada por la escasa frecuencia, por lo cual son necesarias las evidencias alrededor de esta enfermedad.

\section{Asociación entre herpes zóster cutáneo y recaída o progresión del cáncer}

- M. L. Alarcón Tarazona, J. A. Esper Rueda, F. Alzate Amaya, S. A. Higuera Leal, J. E. Fajardo, J. S. Insuasty Enríquez

Objetivo. Determinar la asociación entre la aparición de herpes zóster y posterior recaída o progresión de neoplasia sólida.

Materiales y métodos. Estudio de cohorte retrospectiva en adultos con tumores malignos sólidos tratados en centros de oncología de Bucaramanga entre febrero de 2005 y noviembre de 2011 . Se creó una base de datos en Epi Info 6.04d, se analizó la información en el programa Stata 11. Se determinaron los tiempos libres de progresión y recaída según el tipo de respuesta y exposición, se estimaron los riesgos dados por variables sociodemográficas y clínicas. Se calculó la incidencia de herpes zóster.

Resultados. Se incluyeron 444 pacientes en el grupo de respuesta parcial y 824 en el grupo de respuesta completa. No hubo asociación del herpes zóster con los dos desenlaces de interés: HR para progresión de 0,94 IC95\% 0,61-1,44, recaída de 0,85 IC95\% 0,46-1,55. La mediana del tiempo libre de recaída fue menor en expuestos al zóster cutáneo, 57,1 meses versus 87,4 meses. La incidencia acumulada de zóster cutáneo fue del 6,02\%.

Conclusión. El herpes zóster en pacientes con tumores sólidos malignos no acelera la recaída ni progresión. Se observó una tendencia a la recaída en el grupo de pacientes en respuesta completa expuestos a herpes zóster. La incidencia de herpes zóster corresponde a la reportada en otros estudios. 